\title{
INVESTIGATION OF CELLULAR NETWORK RESOURCE DIVISION PROCEDURES FOR THE JOINT SERVICING OF REAL-TIME MULTISERVICE TRAFFIC AND ELASTIC IOT TRAFFIC
}

\author{
Mukhtar Andrabi Umer, \\ Central University of Kashmir, Srinagar, Jammu and Kashmir, India, umer.andrabi@phystech.edu \\ Sergey N. Stepanov, \\ Moscow Technical University of Communications and Informatics, Moscow, Russia, stpnvsrg@gmail.com
}

DOI: 10.36724/2664-066X-2020-6-I-7-10

\begin{abstract}
Immense growth in the volumes and multiplicity of data to be collected in future Internet of Things (IoT) applications is one of the crucial challenges for the networking organizations as they develop from $4 \mathrm{G}+$ to true $5 \mathrm{G}$ systems. Particularly bulk of this traffic includes complex, unstructured and varied data (Big Data) evolve from smart networking ecosystems (LTE-devices, NB-IoT devices). Although $5 \mathrm{G}$ offers many low power wide area technologies (Lora WAN, GSM and NB-IoT etc.), principally NB-IoT seems very promising addressing the problem because of its certain characteristics like high fault tolerance, delay tolerance, higher coverage area etc. However, due to the limited bandwidth $(180 \mathrm{kHz})$ availability one of the challenges is how to efficiently use these resources to support and handle massive number of growing IoT devices, also resource management and allocation methodology between LTE and NB-IoT traffic flows. In this context, several key issues for IoT communications in $5 \mathrm{G}$ networks should be addressed to satisfy quality of service (QoS) provisioning. In this paper, we propose two scheduling schemes for sharing radio resources between LTE and NB-IoT. The proposed techniques provide scenarios that aims to offer a trade-off between the two types of traffics by guaranteeing the network performance and avoiding unproductive utilization of available resources.
\end{abstract}

KEYWORDS: Narrowband Internet of Things (NB-IoT), Long Term Evolution (LTE), Radio Resource Management (RRM), IoT, LTE Radio Frame Structure.

\section{INTRODUCTION}

Narrowband internet of things (NB_IOT) is one the key technologies in 5th generation cellular networks, due to the fact that this technology has high fault tolerance, delay tolerance, higher coverage area etc. will have a big hand in future communication networks [1].

However, due to the limited bandwidth $(180 \mathrm{kHz})$ availability one of the challenges is how to efficiently use these resources to support and handle massive number of growing IoT. It has been estimated that, by 2020 at least 30 billion devices will be connected to the Internet of Things (Aprox.4 devices / person) and approximately 3.5 billion devices by 2023 [2]. The bulk of the data is expected to be large volumes of heterogeneous, diverse and rapidly changing data referred as Big Data, generated from smart meters, smart sensors, actuators and massive smart video surveillance cameras (Multimedia Big Data) [3]. Such humongous number of devices and IoT applications poses a definite challenge and demand on machine-type or machine-tomachine communication systems, especially Low-Power Wide Area Network (LPWAN) [4].

Considering surveillance and security of prime importance in smart based environments and massive indispensable share of IoT. Smart or intelligent surveillance associates sensing (Smart meters, actuators, and sensors) and video analysis, equates or cross-verifies the inputs and produces precise and accurate results based on heterogeneous data streams. 
For instance, let's consider a scenario where smart meters or sensors deployed on railway tracks detect that a train is over speeding, which can be immediately equated or cross-verified with the video input. As we discussed above, this is going to be a foremost share of the smart IoT ecosystem, and $5 \mathrm{G}$ has structures for merging two or more heterogeneous big data streams for reliable decision making. But the main challenge is resource management and scheduling, while collecting such bulk of heterogeneous big data [5], [6].

While LPWN technologies seems to be the option, especially the evolving Narrow-band Internet of things (NB-IoT) for handling low-end device traffic (meters, sensors etc.), in combination with LTE, for video surveillance. Although there are few characteristics we really need to focus on, while transmitting IoT traffic, which is uplink dominant, especially videos [7], which are event driven in nature and has varying inter-arrival time and constant data size, also exchange large amounts of data after a trigger. This type of data is can be modeled as a possion process [8]. Other includes session management, where session loss and session duration will be strictly balanced for individual data streams for both high rate video cameras and low rate smart meters. Furthermore, balancing of bit rate is also important, by delivering high quality streams at substantially low rates via diverse methods. All these metrics should be as per resource utilization efficiency.

Regrettably, there are very less methodologies proposed by which we can utilize the full potential of $5 \mathrm{G}$ cellular technology for sharing resources between LTE and NB-IoT in mishmash like we discussed above. Also, there are not specific guidelines proposed by 3GPP for such type of resource sharing.

\section{LTE-devices: video cameras}

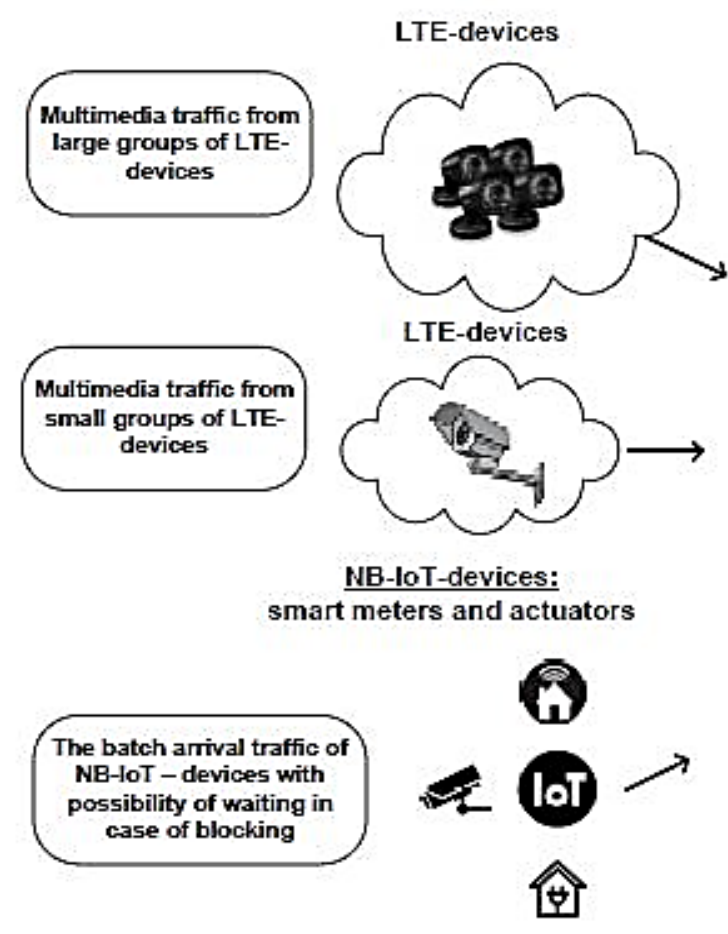

\section{NETWORK MODEL AND ASSUMPTIONS}

The two main radio access technologies LTE Advanced (LTE-A) uses for both Uplink and Downlink are Single-Carrier Frequency Division Access (SCFDMA) and Orthogonal Frequency Division Multiple Access (OFDMA). These two technologies have same radio frame structure and channel subdivision. The channels are divided on the basis of Time and frequency domains.

In the frequency domain, the channel bandwidth ranges from 1 to $20 \mathrm{MHz}$. The full available bandwidth which includes $1.4,3,5,10,15$ and $20 \mathrm{MHz}$ is divided into sub-channels of 12 sub-carriers of $15 \mathrm{KHz}$, totalling $180 \mathrm{KHz}$. The minimum allocation unit of radio resources is called Resource Block (RB). A single RB consists of $180 \mathrm{KHz}$ in the frequency domain and $1 \mathrm{~ms}$ in the time domain. In the time domain, radio resources are divided into Transmission Time Intervals (TTI), also called subframe, with duration of $1 \mathrm{~ms}$.

One frame is formed by 10 TTI. Each TTI consists of two $0.5 \mathrm{~ms}$ slots, and each slot contains seven symbols. LTE-A network within the $5 \mathrm{G}$ environment is considered as a model, it is known as 5G LTE-A.

We focus on a single-cell with one eNB (Base Station) and a set of both LTE and NB-IoT devices, as shown in Fig. 1. There are two types of user traffics: LTE and NBIoT. Assuming that the available bandwidth in the eNB is denoted by $\mathrm{BW}$ and it consists of a number of resource blocks denoted by RBs which is shared between both traffic flows.

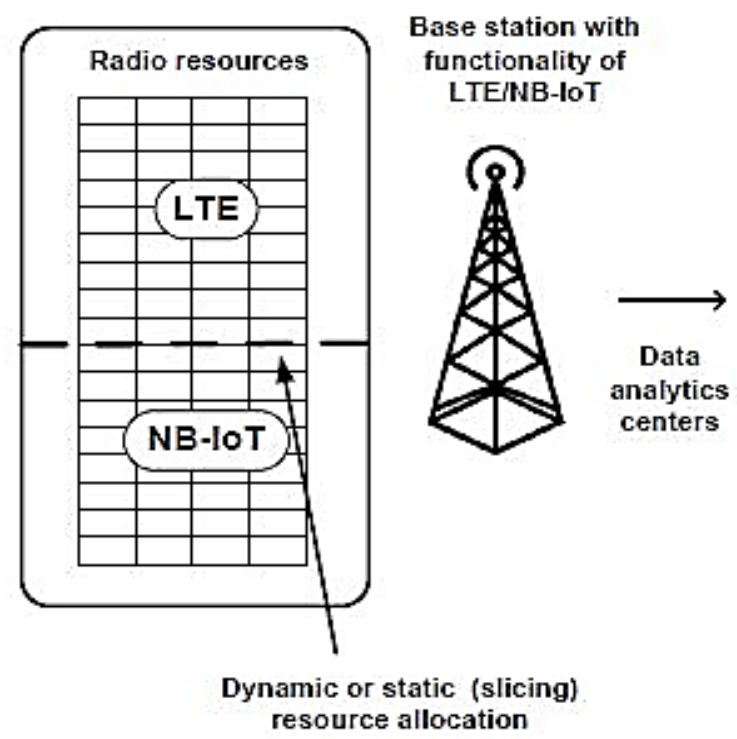

Figure 1. The model of sessions forming by LTE and of NB-IoT devices 


\section{METHODOLOGY}

In order to solve problem, an uplink radio resource management scheme is proposed for the 5G LTE-A system. A single-cell with one eNB is focused on various sets of LTE and NB-IoT equipment. The considered traffic is between e-NB and user equipment. These users can be LTE or NB-IoT. Packets transmission is done between e-NB and users. In this segment, two different schedulers will be discussed: the first one is classic called a Static Scheduler and the second one is with concept of "Renting or Borrowing", called as Dynamic Schedular.

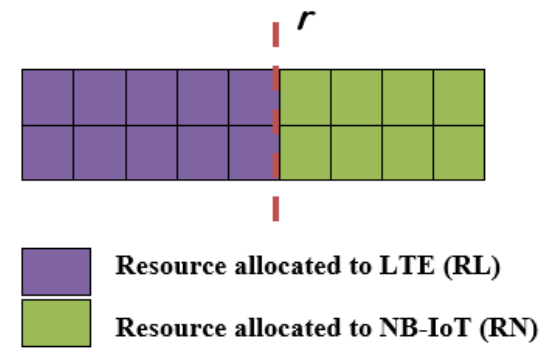

Figure 2. Initialization: Resource blocks assignment at one TTI

The static scheduler has a distribution policy of available resource blocks (in the eNB) between users at one Transmission Time Interval (TTI: division of radio resources in time domain).

The process starts with the initialization step, where random number of resource blocks are chosen and assigned for both LTE and NB-IoT flows as it is shown by the Fig. 2.

In this step, $\mathrm{L}$ and $\mathrm{N}$ denotes the total number of resource blocks designed respectively for LTE and NBIoT.

In order to give priority to NB-IoT flows, a random value $\boldsymbol{r}$ is given between 0.5 and 0.9 ; Therefore, values of $\mathrm{L}$ and $\mathrm{N}$ will be:

$$
L=r \times R B S \text { and } N=(1-r) \times R B S,
$$

where RBs is the total number of resources allocated during 1 TTI.

Firstly, we compute the total number of requested resource blocks designed by ReqL by:

$$
\operatorname{Re} q L=\sum_{i=1}^{k} r^{R B} F i
$$

Where $R e q L$ is total number of requested RBs for (LTE) L- flows, $i \in 1 \mathrm{k}$ is index of L-flows, $\mathrm{k}$ number of L-flows requested and $r^{R \bar{E}} \mathrm{Fl}$ is number of RBs requested for L-flow $i$.

Secondly, we compute the number of $\mathrm{H}-\mathrm{H}$ requested resource blocks designed by Rec $q$ by:

$$
\operatorname{Re} q N=\sum_{j=1}^{h} r^{R B} F j
$$

Where ReqL is total number of requested RBs for (NB-IoT) N- flows, $i \in 1$. $\mathrm{h}$ is index of L-flows, $\mathrm{k}$ number of N-flows requested and $\mu^{n \bar{F}} \mathrm{~F} /$ is number of $\mathrm{RBs}$ requested for $\mathrm{N}-$ flow $j$.

The proposed RRM scheme is summarized by computing the bandwidth consumption rate " $B W c r$ " given by the following equations:

$$
\begin{aligned}
& B W c r=B W c r L+B W c r N \\
& \frac{1}{L R} \sum_{i=1}^{k^{\prime}} r^{R B} F i+\frac{1}{N R} \sum_{j=1}^{h^{\prime}} r^{R B} F j
\end{aligned}
$$

Where $B W c r L$ and $B W c r N$ are band utilization rates for $\mathrm{L}$ and $\mathrm{N}$ flows respectively, where LR and NR are number of RBs designed for $\mathrm{L}$ and $\mathrm{N}$ flows respectively, $k$ ' is number of $\mathrm{L}$ accepted of L-flows and $h^{\prime}$ is number of $\mathrm{N}$ accepted of $\mathrm{N}$-flows.

While in dynamic scheduling, the idea is to maximize the bandwidth utilization rate in the Radio Resource Management (RRM) with Renting or Borrowing system, a dynamic event is added to the static scheduler. By applying the Renting or Borrowing system of resource blocks between LTE and NB-IoT flows with a respect of rules and priorities explained below. In fact, the main idea is to make a random $\boldsymbol{r}$ variant; For example, if LTE flows need more resources, and there are some free resource blocks in NB-IoT area, the LTE flows will Rent or Borrow these resources as shown in Fig. 2. Also, in the static scheduler, each group of flows will be divided into other smaller groups:

Low Priority traffic (LP): flows that does not require resources immediately and can wait.

HP always has the biggest index of priority over LP. This priority is strictly followed in each buffer (LTE and NB-IoT), as shown in Fig. 3.

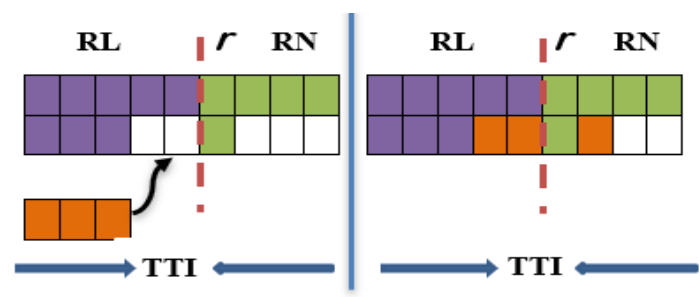

Figure 3. Dynamic borrowing process between RL and RN

he initialization step is almost same in Dynamic schedular as compared to Static, the differences is in Static the resources are distributed evenly and if a high priority process arrives in a specified area (LTE or NBIoT), these are made to wait until the resource is available. While in Dynamic scheduling if the resources are not enough to serve a particular flow, we compute the allotted RBs in both areas and if there is availability, these are rented or barrowed.

Compared to the static scheduler, we have the same beginning in the dynamic scheduler same initialization phase and the same start TTI architecture 


$$
\begin{aligned}
& P A s g R B_{L}=\sum_{i=1}^{k^{\prime}} P A c k^{R B} F i \\
& P A s g R B_{N}=\sum_{j=1}^{h^{\prime}} P A c k^{R B} F j
\end{aligned}
$$

Where $P A g g R B_{L}$ and $P A g g R B_{W}$ are number of RBs pre-assigned to $\mathrm{L}$ and $\mathrm{N}$ flows respectively, while PAck ${ }^{A D} \mathrm{~F}$ a acknowledged RBs for $\mathrm{L}$ and $\mathrm{N}$ flows.

When the available resource blocks are not enough to satisfy $R e q L$ requests, we compute the remained RBs for $\mathbf{N}$ (NB-IoT) and in the other case we compute the remained RB for $\mathbf{L}$ (LTE) as given below

$$
\begin{aligned}
& R \operatorname{Req} L=L R-\text { AllocR }_{L} \\
& R \operatorname{Re} q N=N R-\text { AllocR }_{N}
\end{aligned}
$$

Where RReqL and $R R$ eqAF are number of RBs remained for $\mathrm{L}$ and $\mathrm{N}$ flows, while Alloc $R B_{L}$ and Alloc $R B_{W}$ represent number of pre-allocated RBs for respective flows.

Afterwards, we calculate the number of not served flows in each branch if it exists, $p=k-k^{\prime}$ _for L-flows and $q=h-h$ ' for N-flows. Then, we calculate, in each branch respectively, the functions

$$
\begin{aligned}
& \text { ReqL }- \text { AllocR }_{L} \leq N-\text { AllocRB }_{N} \\
& \operatorname{Re} q N-\text { AllocR }_{N} \leq L-\text { AllocRB }_{L}
\end{aligned}
$$

Where $\mathrm{p}$ and $\mathrm{q}$ represent number of non-served $\mathrm{L}$ and $\mathrm{N}$ flows.

If these equations are verified and the RBs are enough, all flows are accepted and allocated. We have spoken previously about priority of the $\mathbf{L}$ flows and we will see now the effect of this prioritization: - We admit $h$ "'- preadmitted flows and allocate the resource blocks for the $h$ "-flows, calculated as in the below equation, reject the $q$ _ flows and compute the $\mathbf{N}$ utilization rate

$$
A l c R B_{N}=\sum_{j=1}^{h "} A d m i t^{R B} F j
$$

Where $A l l R B_{W}$ is number allocated RBs to N-Flows $h^{r s}$, number of pre-admitted N-flows, Admit ${ }^{R E}$ Fy denote number of admitted RBs for $\mathrm{N}$-flow $\mathbf{F}$ j.

\section{CONCLUSION}

In this paper firstly we discussed how NB-IoT has a huge role to play in the sphere of Internet of things and how this technology can be used to address the problem of growing data and devices tremendously in our communication networks. Also, we discussed two schedulers for IoT communications based on QoS requirements of LTE and NB-IoT flows. The proposed schemes give best scenarios that aim to provide a tradeoff between the two types of traffics by guaranteeing the network performance and avoiding ineffective exploitation of available resources.

\section{REFERENCES}

1. Introductio to NB-IoT, document RP-161248, 3GPP, Ericson, Nokia, ZTE, NTT DOCOMO Inc. Busan, South Korea, 2016.

2. Huawei NB-IoT - Enabling New Business Opportunities, Technical Report, 2015.

3. S. Step ov, M. Stepanov, A. Tsogbadrakh, J. Ndayikunda and U. Andrabi. Resource Allocation and Sharing for Transmission of Batched NB IoT Traffic Over 3GPP LTE. 2019 24th Conference of Open Innovations Association (FRUCT), Moscow, Russia, 2019. P. 422-429. doi: 10.23919/FRUCT.2019.8711920.

4. A. Hoglu et al. Overview of $3 \mathrm{GPP}$ release 14 enhanced NB-IoT. IEEE Netw. 2017. Vol. 31. No. 6. P. 16-22.

5. H. Malik, H. Pervaiz, M. Mahtab Alam, Y. Le Moullec, A. Kuusik and M. Ali Imran. Radio Resource Management Scheme in NB-IoT Systems. IEEE Access. 2018. Vol. 6. P. 15051-15064. doi: 10.1109/ACCESS.2018.2812299.

6. V. Begishev, V. Petrov, A. Samuylov, D. Moltchanov, S. Andreev, Y. Koucheryavy, K. Samouylov. Resource Allocation and Sharing for Heterogeneous Data Collection over Conventional 3GPP LTE and Emerging NB-IoT Technologies. Computer Communications. 2018. Vol. 120. No. 2. P. 93-101.

7. N. Nikaein e al. Simple traffic modeling framework for machine type communication. 2013. P. 1-5.

8. Wang, iaoli \& Sheng, MingJye \& Lou, Yuan-Yao \& Shih, Yuan-Yao \& Chiang, Mung. Internet of Things Session Management over LTE - Balancing Signal Load, Power and Delay. IEEE Internet of Things Journal. 2016. No. 3. 1-1. doi: 10.1109/JIOT.2015.2497230. 